\title{
Comprehensive Review of AL amyloidosis: some practical recommendations
}

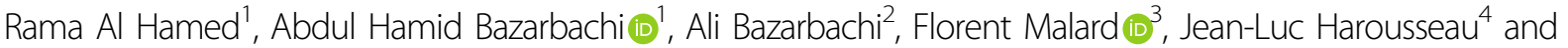 \\ Mohamad Mohty (1) ${ }^{3}$
}

\begin{abstract}
Amyloid light chain (AL) amyloidosis is among the more common and more severe of the amyloidoses usually involving the slow proliferation of a bone-marrow-residing plasma cell (PC) clone and the secretion of unstable immunoglobulin-free light chains (FLC) that infiltrate peripheral tissues and result in detrimental end-organ damage. Disease presentation is rather vague, and the hallmark of treatment is early diagnosis before irreversible end-organ damage. Once diagnosed, treatment decision is transplant-driven whereby $\sim 20 \%$ of patients are eligible for autologous stem cell transplantation (ASCT) with or without bortezomib-based induction. In the setting of ASCTineligibility, bortezomib plays a central role in upfront treatment with the recent addition of daratumumab to the current emerging standard of care. In general, management of AL amyloidosis is aimed at achieving deep, durable responses with very close monitoring for early detection of relapse/refractory disease. This article provides a comprehensive review of the management of patients with AL amyloidosis including goals of therapy, current treatment guidelines in the setting of both ASCT-eligibility and ineligibility, treatment response monitoring recommendations, toxicity management, and treatment of relapse/refractory disease.
\end{abstract}

\section{Introduction}

Systemic amyloidoses constitute disorders of diverse etiologies involving the synthesis and abnormal extracellular deposition of misfolded proteins in various organs with resultant damage. Among the different amyloidoses, amyloid light chain (AL), previously called "primary", amyloidosis is among the more common and more severe, affecting $\sim 10$ per million per year ${ }^{1}$. Even though rarely due to a nonplasma cell $\mathrm{B}$-cell close, $\mathrm{AL}$ amyloidosis is usually a plasma cell (PC) disorder whereby a small, slowly proliferating, bone-marrow-residing PC clone, secretes unstable immunoglobulin light chains. Those amyloidogenic free light chains (FLCs) can then infiltrate peripheral organs resulting in organ dysfunction and

Correspondence: Mohamad Mohty (mohamad.mohty@inserm.fr)

${ }^{1}$ Department of Internal Medicine, Jacobi Medical Center, Albert Einstein College of Medicine, New York, NY, USA

${ }^{2}$ Department of Internal Medicine, American University of Beirut, Beirut, Lebanon

Full list of author information is available at the end of the article ultimately, failure. Organs involved usually include kidneys, heart, gastrointestinal (GI) tract, liver, and nervous system whereby undoubtedly cardiac involvement is the main driver of disease prognosis and mortality ${ }^{2}$. Given that more than $69 \%$ of patients already have more than one organ involved at the time of diagnosis ${ }^{3}$, it becomes vital to not only diagnose AL amyloidosis early, but to also effectively control plasma cell dyscrasias and thus halt escalating organ damage.

Owing to toxicity, autologous stem cell transplantation (ASCT) remains the standard of care and first-line treatment in a small proportion of patients. Importantly, the outcome of ASCT-ineligible patients has improved by the introduction of new first-line agents, specifically bortezomib. Currently, active research involving second-line novel treatments and anti-CD38 antibodies offers new hope. Nonetheless, the main hallmark of management remains early recognition and initiation of treatment before the occurrence of irreversible organ damage.

\section{(c) The Author(s) 2021}

(c) (i) Open Access This article is licensed under a Creative Commons Attribution 4.0 International License, which permits use, sharing, adaptation, distribution and reproduction c. in any medium or format, as long as you give appropriate credit to the original author(s) and the source, provide a link to the Creative Commons license, and indicate if changes were made. The images or other third party material in this article are included in the article's Creative Commons license, unless indicated otherwise in a credit line to the material. If material is not included in the article's Creative Commons license and your intended use is not permitted by statutory regulation or exceeds the permitted use, you will need to obtain permission directly from the copyright holder. To view a copy of this license, visit http://creativecommons.org/licenses/by/4.0/. 
It becomes vital to differentiate $\mathrm{AL}$ amyloidosis from transthyretin (TTR) amyloidosis, another disease of increasing prevalence, due to the difference in management. TTR is a liver-synthesized thyroxine and vitamin A transporter ${ }^{4,5}$. Age (wild-type, previously called senile amyloidosis) or an autosomal dominant amino acid substitution (mutant) result in fibrillogenesis whereby TTR dissociates into intermediates that misassemble into amyloid fibrils and deposit in end-organs, most notably the heart ${ }^{6}$. While AL amyloidosis is treated with chemotherapy and transplant, TTR cardiac amyloidosis is treated with targeted therapy such as tafamidis ${ }^{7}$.

\section{Diagnostic approach}

The symptoms and the presentation of amyloidosis depend on the organs involved in the disease and so, patients can present with a myriad of unspecific symptoms that could be easily misinterpreted, thus clouding the diagnosis of amyloidosis and delaying treatment initiation. Common symptom constellations include, but are not limited to, heart failure with preserved ejection fraction (HFpEF), nephrotic range proteinuria, organomegaly due to amyloid deposition (hepatomegaly, macroglossia, enlarged salivary glands etc.), peripheral neuropathy, and constitutional symptoms (weight loss, fatigue) (Fig. 1). By the time such symptoms surface, organ damage has already occurred. It thus becomes important to identify high-risk patient populations who would benefit from regular screening to establish a diagnosis before symptom onset. This includes monoclonal gammopathy of undetermined significance (MGUS) patients who would benefit from regular followup of markers such as $\mathrm{N}$-terminal pro-brain natriuretic peptide (NT-proBNP) and albuminuria, which could begin to rise before overt HF and nephrotic syndrome develop $^{8,9}$ (Fig. 1). Once suspected and a monoclonal component is confirmed, confirmation requires tissue biopsy (fat pad, bone marrow (BM), salivary gland, involved organ) and typing (mass spectrometry [current gold standard], immunogold electron microscopy, immunofluorescence, and immunohistochemistry) followed by risk stratification and disease staging ${ }^{9}$ (Fig. 1).

There are four staging models that utilize cardiac soluble biomarkers-Mayo 2004 model, European 2015 modification of Mayo 2004 model, Mayo 2012 model, and Boston University (BU) model ${ }^{10-14}$ (Table 1 ). The Mayo 2004 model, uses troponin T (TnT) and NT-proBNP and categorizes disease into one of three stages according to the specified thresholds of TnT $<; 0.035$ microg/L and NTproBNP $<332 \mathrm{ng} / \mathrm{L}^{11}$. Depending on whether $\mathrm{TnT}$ and NT-proBNP were both low, only one was high or both were elevated, disease was classified as stage I, II, or III, respectively ${ }^{11}$. In 2015, a European group proposed a modification of the Mayo 2004 model, in which patients classified in stage III of the Mayo model were further subclassified into low-risk and high-risk groups, stage IIIA and IIIB, using a new threshold for NT-proBNP of $8500 \mathrm{ng} / \mathrm{L}$. Patients with an NT-proBNP $>8500 \mathrm{ng} / \mathrm{L}$ had

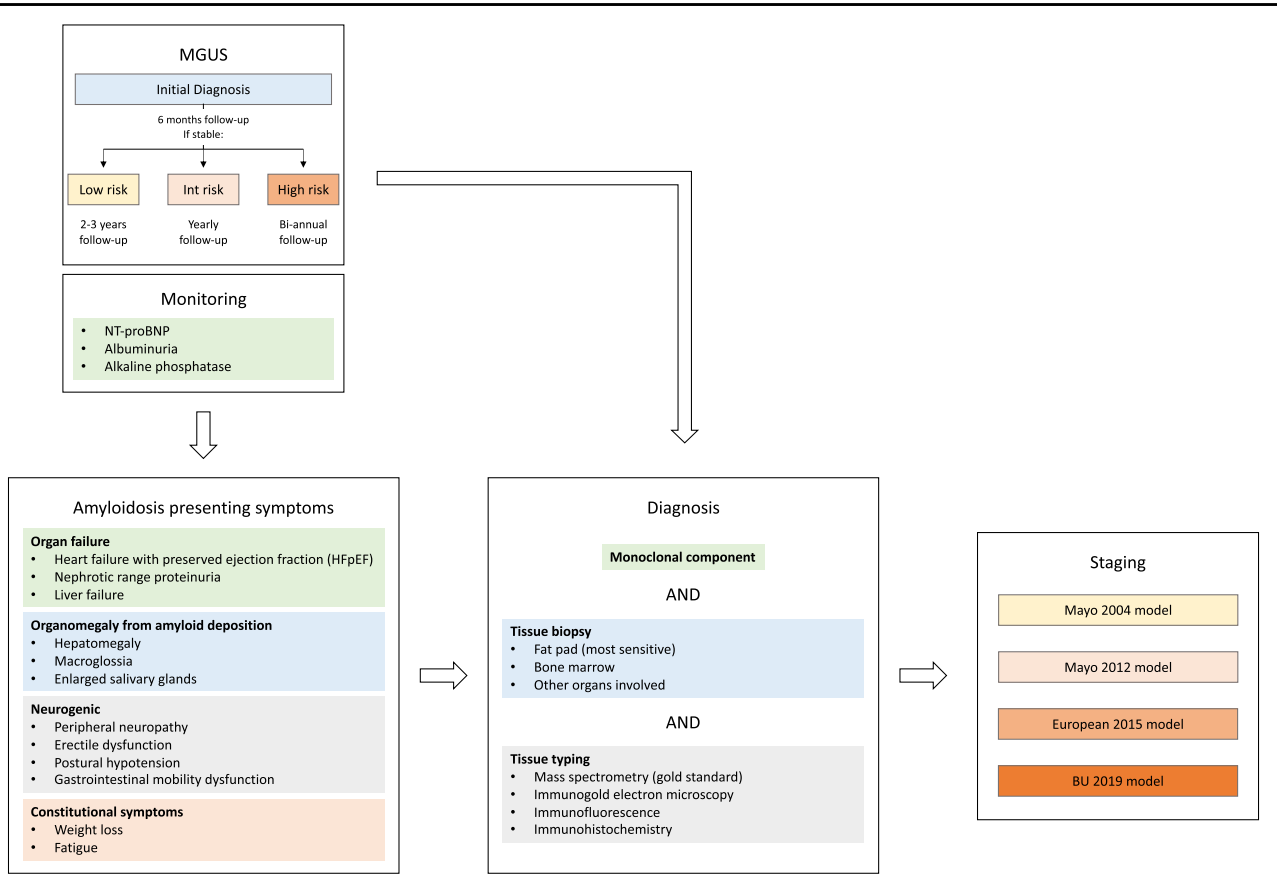

Fig. 1 Monitoring, presenting symptoms, anddiagnosis of AL amyloidosis. MGUS monoclonal gammopathy of undetermined significance, NT-proBNP N-terminal pro b-type natriuretic peptide. 
Table 1 Amyloidosis staging models.

\begin{tabular}{|c|c|c|c|c|}
\hline Model & Criteria & Points & Score & Stage \\
\hline \multirow[t]{3}{*}{ Mayo 2004} & Troponin $(\operatorname{TnT})>0.035$ microg $/ \mathrm{L}$ & 1 point & 0 & Stage I \\
\hline & & & 1 & Stage II \\
\hline & NT-proBNP > 332 ng/L & 1 point & 2 & Stage III \\
\hline \multirow[t]{4}{*}{ Mayo 2012} & Troponin $(T n T) \geq 0.025 \mathrm{ng} / \mathrm{mL}$ & 1 point & 0 & Stage I \\
\hline & & & 1 & Stage II \\
\hline & NT-proBNP $\geq 1800$ pg/mL & 1 point & 2 & Stage III \\
\hline & $\mathrm{dFLC} \geq 18 \mathrm{mg} / \mathrm{dL}$ & 1 point & 3 & Stage IV \\
\hline \multirow[t]{6}{*}{ European 2015} & Troponin $(\operatorname{TnT})>0.035$ microg $/ \mathrm{L}$ & 1 point & 0 & Stage I \\
\hline & & & 1 & Stage II \\
\hline & NT-proBNP > 332 ng/L & 1 point & & \\
\hline & & & 2 & Stage IIIa \\
\hline & NT-proBNP > 8500 ng/L & 1 additional point if score $=2$ & & \\
\hline & & & 3 & Stage IIIb \\
\hline \multirow[t]{6}{*}{ BU 2019} & Troponin $(T n l) \geq 0.1 \mathrm{ng} / \mathrm{mL}$ & 1 point & 0 & Stage I \\
\hline & & & 1 & Stage ॥ \\
\hline & $\mathrm{BNP} \geq 81 \mathrm{pg} / \mathrm{mL}$ & 1 point & & \\
\hline & & & 2 & Stage III \\
\hline & $\mathrm{BNP} \geq 700 \mathrm{pg} / \mathrm{mL}$ & 1 additional point if score $=2$ & & \\
\hline & & & 3 & Stage IIIb \\
\hline
\end{tabular}

higher risk disease and poorer prognosis ${ }^{12}$. The Mayo model was revised in 2012 incorporating different thresholds for TnT and NT-proBNP and introducing difference in FLC (dFLC) as an additional marker for disease burden ${ }^{13}$. One point was attributed to each of TnT $\geq 0.025 \mathrm{ng} / \mathrm{mL}, \quad \mathrm{NT}$-proBNP $\geq 1800 \mathrm{pg} / \mathrm{mL}$, and $\mathrm{dFLC} \geq 18 \mathrm{mg} / \mathrm{dL}$, thus classifying disease into four stages, I-IV according to a total score of $0-3$, respectively ${ }^{13}$. Recently, the BU group derived a new staging system that correlates with the Mayo 2004 system, but utilizes BNP instead $^{14}$. A BNP threshold $>81 \mathrm{pg} / \mathrm{mL}$ best identified cardiac involvement and thus correlated with the Mayo 2004 staging system $(\kappa=0.854)^{14}$. Three stages were thus developed based on a BNP $>81 \mathrm{pg} / \mathrm{mL}$ and troponin I $(\mathrm{TnI})>0.1 \mathrm{ng} / \mathrm{mL}$, whereby disease was classified into stages I, II, and III when both markers were lower than prespecified thresholds, only one was elevated or both were elevated, respectively ${ }^{14}$. Furthermore, stage III was further divided to include stage IIIb in the event of a BNP $>700 \mathrm{pg} / \mathrm{mL}^{14}$. This allows for centers without access to NT-proBNP or TnT to accurately stage AL amyloidosis. It is evident that the aforementioned models are very successful in dividing patients into distinct survival groups when examining the 12-year survival curves for the same patient dataset ${ }^{10}$.

\section{Risk-adapted management approach}

When choosing the appropriate treatment approach, potential organ impairment needs to be considered. While the younger and fitter patient can tolerate more intense therapy, the frailer one has poor treatment tolerance and is, as such, at an increased risk of early mortality. The determination of frailty in amyloidosis is not accompanied by specific frailty scores, but short-term survival can be used as a surrogate to estimate frailty. Age and the number, type and extent of organ involvement and to a lesser extent, pre-existing comorbidities, are the main determinants of frailty in this patient population.

The Mayo Clinic investigators looked at the effect of age, among other factors, on survival in a cohort of 592 patients with mass-spectrometry-verified AL amyloidosis between 2008 and 2015. The cohort was followed up for 8 years and the median overall survival (OS) was 44 months. Dividing the patients into two age groups, $<65$ years and $\geq 65$ years, it was evident that the younger patients had a much better survival than those aged $\geq 65$ years ${ }^{15}$.

The effect of the number of organs involved on survival was investigated in the same cohort. There was a significant decrease in survival as the number of organs involved increased $(p<0.001)^{15}$. However, it is not just the number of organs involved in the disease, but also the 
type of organs involved that determine survival. For instance, comparing survival in patients with isolated cardiac involvement to those with cardiac plus multiorgan involvement, yields very similar survival curves between the two groups $(p=0.51)^{15}$. On the other hand, comparing survival in patients with isolated renal involvement to those with renal plus multi-organ involvement, yields significantly different survival curves $(p<0.001)$ whereby isolated renal involvement infers a survival advantage compared to the latter. As such, cardiac involvement is therefore the most important prognostic factor in AL amyloidosis whereby when present, irrespective of other organ involvement, influences survival. As previously discussed, several powerful staging models build on cardiac involvement to classify disease and aid treatment decisions.

Another important determinant of outcome and treatment response is cytogenetic profile. A recent retrospective chart review of $140 \mathrm{AL}$ amyloidosis patients explored the most frequent cytogenetic abnormalities and their impact on survival ${ }^{16}$. Sixty-one percent of the patients harbored a cytogenetic abnormality, the most common of which was translocation $t(11 ; 14)$ accounting for $44 \%$, followed by hyperdiploidy $(43 \%)^{16}$. A statistically significant relationship was noted between several abnormalities and increased plasma cell (PC) burden, including gain $(+) 5 \mathrm{p} / 5 \mathrm{q}(p=0.025)$, del13q $(0.009)$, $+11 \mathrm{q}(p<0.001)$, and hyperdiploidy $(p<0.001)^{16}$. In multivariable analysis, hyperdiploidy was confirmed a significant poor prognostic factor ${ }^{16}$. In the setting of cardiac involvement, hyperdiploidy was also associated with worsening progression-free survival (PFS) $(p=$ $0.0497)$ and OS $(p=0.006)^{16}$. Similarly, del13q was associated with cardiac involvement but did not appear to impact survival ${ }^{16}$. In addition, the overall presence of $t(11 ; 14)$ did not carry any prognostic value in terms of PFS and OS, although on further stratification, patients harboring isolated $t(11 ; 14)$ had relatively worse PFS relative to those without any cytogenetic abnormalities ${ }^{16}$. Conversely, the absence of any cytogenetic abnormalities was associated with improved PFS and OS $(p=0.042$ and 0.019 , respectively ${ }^{16}$. This becomes important as well when choosing therapy. For instance, patients with $+1 \mathrm{q}$ were found to have a better response to treatment with daratumumab, a monoclonal receiving increased attention in the field ${ }^{16}$. As will be further discussed, patients harboring $t(11 ; 14)$ are more likely to have disease resistant to bortezomib-based regimens and are more likely to respond to venetoclax ${ }^{17,18}$.

\section{Treatment of ASCT-eligible patients}

The first question to answer upon considering a treatment plan for a patient is whether or not the patient is eligible for ASCT. High-dose melphalan with subsequent
ASCT (HDM-ASCT) remains the standard of care in lowrisk patients ${ }^{19}$. Approximately $20 \%$ of patients are likely eligible for this procedure. For those ineligible, a cautious approach is necessary, involving standard intensity and, for the frailer patients, low-intensity therapies (Fig. 2).

The eligibility criteria for ASCT have evolved over the years and with the emergence of novel agents ${ }^{12,20-22}$. Typically, potentially reversible contraindications are assessed before deeming a patient ineligible for ASCT. Otherwise, the presence of one exclusion criteria, specifically pertaining to cardiopulmonary or renal status could suffice in making a patient ASCT-ineligible. The most common eligibility criteria for ASCT are?

- Age $<70$ years

- Eastern Cooperative Oncology Group (ECOG) performance status $<2$

- NYHA Class < III (New York Heart Association classification of the extent of heart failure)

- Left ventricular ejection fraction (LVEF) $>45 \%$

- Systolic blood pressure (SBP) $\geq 100 \mathrm{mmHg}$

- $\mathrm{TnT}<0.06 \mathrm{ng} / \mathrm{mL}$ or high-sensitivity (hs) $-\mathrm{TnT}<$ $75 \mathrm{ng} / \mathrm{mL}$

- NT-proBNP < $5000 \mathrm{ng} / \mathrm{L}$

- Creatinine clearance $(\mathrm{CrCl})>50 \mathrm{ml} / \mathrm{min}$ (unless on chronic dialysis)

- Bilirubin $<2 \mathrm{mg} / \mathrm{dL}$

- Diffusion capacity of the lungs for carbon monoxide (DLCO) $>50 \%$

- No more than two organs significantly involved

The choice of therapy for ASCT-eligible patients has evolved over time and has been a matter of perspective for many years. The advantages of stem cell transplantation are high response rate and response durability whereas the disadvantages are the short-term morbidity and mortality associated with it, which can be quite significant, and the fact that this therapy can only be offered to a minority of select patients. Nonetheless, complete response (CR) rates achieved with ASCT remain higher than those possible with any other treatment regimen but data from two major groups show that CR rates after ASCT are conditioningdose dependent ${ }^{23,24}$. A study carried out between July 1994 and December 2008 compared HDM ( $\left.200 \mathrm{mg} / \mathrm{m}^{2}\right)$ to a reduced dose $\left(100-140 \mathrm{mg} / \mathrm{m}^{2}\right)$ in $421 \mathrm{AL}$ amyloidosis patients. The results demonstrated a significantly higher $\mathrm{CR}$ rate in the high-dose group (43\% versus $24 \%, p<$ $0.001)^{24}$. Similarly, between January 2000 and August 2015 , a study of 457 patients using the same melphalan conditioning-dose levels, showed a significantly higher CR rate in the high-dose group (53\% versus $37 \%, p=0.003)^{23}$. In both studies, high-dose melphalan was associated with lower transplant-related mortality (TRM) (9\% versus $14 \%$, $p=0.12 ; 2 \%$ versus $6 \%, p=0.01)^{23,24}$. The median PFS and OS were also significantly longer in the high-dose groups in both studies. 


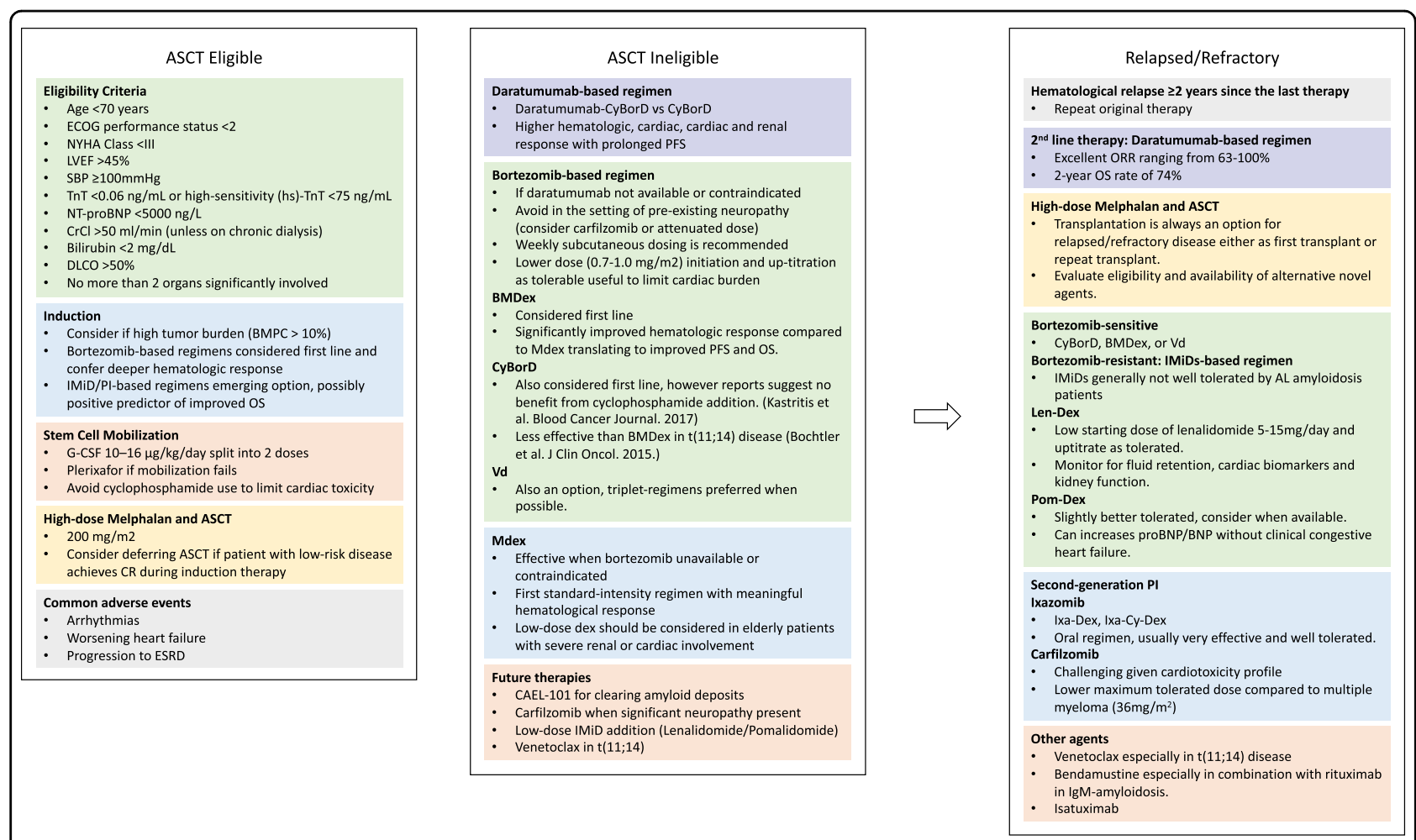

Fig. 2 Treatment algorithm for AL amyloidosis. ASCT autologous stem cell transplantation, ECOG Eastern Cooperative Oncology Group, NYHA New York Heart Association classification of the extent of heart failure, LVEF left ventricular ejection fraction, SBP systolic blood pressure, TnT troponin $\mathrm{T}, \mathrm{CrCl}$ creatinine clearance, DLCO diffusion capacity of the lungs for carbon monoxide, BMPC bone marrow plasmacytosis, IMiD immunomodulatory imide drugs, PI proteosome inhibitors, OS overall survival, G-CSF granulocyte colony-stimulating factor, CR complete remission, PFS progression-free survival, ESRD end-stage renal disease, ORR overall response rate.

Despite the deeper responses achieved with ASCT compared to other treatment modalities, it is important to assess the durability of such a response. A recent study looking into the need for subsequent or second-line therapy after ASCT in 186 patients highlighted that in those who had survived for at least 10 years post diagnosis, $47 \%$ remained treatment-free ${ }^{25}$. When categorized according to initial treatment modality, ASCT treatment versus standard-intensity therapies, $58 \%$ of ASCT patients were treatment-free at 10 years compared with only $36 \%$ in the non-ASCT group $(\Delta 22 \%)^{25}$. As such, long-term survivors are increasingly seen in AL amyloidosis and ASCT is associated with a more durable response compared with standard-intensity therapies.

That said, a randomized controlled trial in 100 patients performed between 2000 and 2005 by the Intergroupe Francophone du Myélome (IFM) reported evidence that argued against ASCT for AL amyloidosis ${ }^{26}$. It compared high-dose standard-intensity therapy (melphalan + dexamethasone) with HDM and ASCT rescue. The nonASCT arm had an improved survival $(p=0.04)^{26}$. Although HDM followed by ASCT had been in use for more than a decade by then, some of the participating centers in the study had just started adopting HDM-
ASCT as a treatment strategy, thus contributing to the study's limitation. In addition, patient selection criteria were not well established. For example, of the 50 patients randomized to the HDM-ASCT arm, only 37 (74\%) went on to receive ASCT. This translated into a high day +100 TRM of $24 \%$ in this group ${ }^{26}$. Most of the remaining patients went on to have an early death ${ }^{26}$. Along the same lines, a systematic review of 12 studies also found no advantage of ASCT over conventional chemotherapy in improving OS in $\mathrm{AL}$ amyloidosis ${ }^{27}$. However, the evidence was weak, and the authors cautioned that further research was needed ${ }^{27}$.

In North America between 1995 and 2012, a randomized, multicenter study of $1536 \mathrm{AL}$ amyloidosis patients at 134 centers, demonstrated that the rate of early mortality after ASCT at 30 days and 100 days progressively declined over successive time periods (1995-2000, 2001-2006, and $2007-2012)^{28}$. At 30 days, the TRM rates were $10 \%, 7 \%$, and $4 \%$ for these time periods, respectively, and at 100 days $19 \%$, $11 \%$, and $4 \%$, respectively, $(p<0.01)^{28}$. Also, centers performing more than four transplantations per year for AL amyloidosis had superior survival outcomes ${ }^{28}$. This data highlight the importance of center experience in achieving better outcomes in this patient population but also explain 
one of the potential limitations of the IFM study-relatively earlier time of investigation.

\section{Induction chemotherapy}

Another controversial aspect of ASCT in AL amyloidosis is whether or not induction chemotherapy is required. Hwa et al. investigated the impact of induction chemotherapy on the response following transplant ${ }^{29}$. All patients $(n=415)$ who received ASCT within 12 months of diagnosis were included and were divided according to baseline bone marrow plasmacytosis (BMPC) level into two groups $(>10 \%, n=116 \text { versus } \leq 10 \%, n=299)^{29}$. Patients with a low tumor burden $(\mathrm{BMPC} \leq 10 \%)$ did not show any difference in response rate irrespective of induction chemotherapy ${ }^{29}$. For patients with BMPC > $10 \%$, the results were very different though, whereby the overall response rate (ORR) was significantly better with a near doubling of the complete response (CR) rate in patients who had received induction therapy pre-ASCT (34\% versus $18 \%, p=0.048)^{29}$. Afrough et al. highlighted the improved survival with pre-ASCT induction as well in 128 patients comparing conventional induction with melphalan or steroids to novel agents such as the thalidomide-analogues, the immunomodulatory imide drugs (IMiDs), and proteosome inhibitors (PI) whereby hematological, but not organ response, was significantly higher with IMiD/PI-based regimens ${ }^{30}$. Induction with IMiD/PIbased regimens was also found to be a significant positive predictor of $\mathrm{OS}^{30}$.

Multiple clinical trials have also looked into the role of bortezomib or bortezomib-based regimens in both induction and conditioning. A prospective, single-arm trial (NCT01083316) used bortezomib-dexamethasone (Vd) for induction followed by bortezomib-HDM for conditioning whereby $100 \%(n=27)$ achieved hematological response (HR) with $63 \% \mathrm{CR}$ and $37 \%$ very good partial response (VGPR) 6 months post-ASCT ${ }^{31}$. The cohort of patients was followed up for an extended median of 77 months whereby renal and cardiac responses occurred in $65 \%$ and $88 \%$, respectively, at 5 -years post$\mathrm{ASCT}^{31}$. Median OS and PFS were not yet reached highlighting the durability of response with the incorporation of bortezomib ${ }^{32}$. The phase II HOVON 104 trial (NTR3220) investigated the use of four cycles of $\mathrm{Vd}$ induction treatment, followed by HDM-ASCT ${ }^{33}$. The overall $\mathrm{HR}$ after induction was $80 \%$ including $20 \% \mathrm{CR}$ and $38 \%$ VGPR with improvement 6 months post-ASCT to overall HR of $86 \%$ with $46 \%$ CR and $26 \%$ VGPR. The trial confirmed the efficacy of $\mathrm{Vd}$ and demonstrated the amplified outcome post-ASCT. However, due to treatment-related toxicity and disease characteristics, primary endpoint could not be reached ${ }^{33}$.

It remains relatively unclear whether $\mathrm{CR}$ in response to induction alone is comparative to $\mathrm{CR}$ in response to induction followed by ASCT. Given the morbidity and mortality associated with transplantation, it is probably best to wait and observe rather than proceed to ASCT in a patient who has achieved $C R$ in response to induction chemotherapy. At this point though, stem cells could be collected for future ASCT. On the other hand, in patients who achieve CR with induction chemotherapy but have a higher chance of early relapse, proceeding to ASCT may be the better option if they are eligible for it. These may be patients who have high-risk fluorescence in situ hybridization (FISH) genetics (uncommon in amyloidosis patients), such as those previously discussed, or patients with very high BMPC at diagnosis, which resembles multiple myeloma (MM) with amyloidosis.

Given the rapid progression of amyloidosis and the fact that diagnosis and subsequent treatment are a race against the clock, it is imperative that patients do not suffer further deterioration in organ function. As such, induction chemotherapy with bortezomib-based regimen should be considered for stem cell eligible patients, particularly with $\mathrm{BMPC}>10 \%$ or in the event of a foreseeable delay of $>1$ month in ASCT $^{9}$.

\section{ASCT-associated toxicity}

The first stage in the management of toxicity in relation to ASCT is during stem cell mobilization and collection whereby low serum albumin, elevated NT-proBNP and increased septal thickening were found to be important risk factors for toxicity ${ }^{34,35}$ During stem cell mobilization, patients can experience various toxicities including tachyarrhythmias, thromboembolic events, weight gain (due to fluid retention), bleeding, acute kidney injury, hypertensive crises, or hypotension. To minimize risk of toxicity, it is recommended to use granulocyte-colonystimulating factor (G-CSF) without cyclophosphamide given the increased cardiac morbidity, significantly higher number of apheresis required, increased hospitalizations and increased toxicity associated with the latter ${ }^{36}$. The recommended dose of G-CSF is $10-16 \mu \mathrm{g} / \mathrm{kg} /$ day, either as one dose or divided into two doses, 3 days before stem cell collection for an optimal total of at least $5 \times 10^{6} \mathrm{CD} 34+$ cells $/ \mathrm{kg}^{37}$. The general recommendation though, is to split the dose. If patients fail stem cell mobilization, plerixafor is a well-tolerated adjuvant ${ }^{36}$.

The major ASCT toxicities remain cardiac arrhythmias, worsening HF, syncope, and end-stage renal disease (ESRD). Twenty-five percent of patients who have had pretransplant 24-h Holter monitoring will have evidence of nonsustained ventricular tachycardia (NSVT), a condition associated with an inferior short-term (6 months) survival $^{38}$. On adjustment for cardiac (Mayo) stage, NSVT was actually not found to impact peritransplant mortality and pretransplant NSVT is therefore not an exclusion criterion $^{13,38}$. As such, $\sim 50 \%$ of patients will experience 
arrhythmias following ASCT, most of which will be low grade but with grade 3/5 arrhythmias accounting for $\sim 10 \%{ }^{39}$. It remains unclear whether primary AICD (Automatic Implantable Cardioverter Defibrillator) implantation will be of benefit, even among those who are at high-risk of sudden cardiac death and so, decisions should be case specific.

Worsening heart failure is another toxicity that may be encountered during transplantation and about 5\% of patients will have a reduction in EF of $>10 \%$, which increases the 100-day mortality ${ }^{40}$. Multiple factors contribute to the heart failure risk during peritransplant in this population including electrolyte imbalance, medications, fluid overload, arrhythmia, temporary cessation of cardiac medications, sepsis, and exacerbation of organ dysfunction during transplant. It is worth noting that standard heart failure treatments such as beta blockers, calcium channel blockers and/or ACE inhibitors, cannot be used for worsening heart failure management in this patient population and so should be avoided ${ }^{41}$.

Another important toxicity that could complicate ASCT is ESRD. In a study examining the association between acute renal failure and mortality in AL amyloidosis during ASCT, the medical records of 408 ASCT patients between 1996 and 2010 were examined ${ }^{42}$. Dialysis was required by $72(18 \%)$ patients. Eight patients started dialysis $>30$ days prior to ASCT (Group II), 36 started \pm 30 days after ASCT (Group III) and 28 initiated dialysis $>1$ month after ASCT (Group IV) whereby patients who were never dialyzed were assigned to Group I ${ }^{42}$. Median OS was not reached in Groups I and II but was 7 months in Group III and 48.5 months in Group IV $(p<0.001)^{42}$. TRM was observed in $44.4 \%$ of the patients in Group III, 6-fold higher than the next highest group with a TRM of only $3.6 \%(p<0.001)^{42}$. The most common causes of TRM were cardiac and sepsis. In the multivariate analysis, only hypoalbuminemia $(<2.5 \mathrm{~g} / \mathrm{dL}, p<0.001)$ and estimated glomerular filtration rate (eGFR) $<40 \mathrm{~mL} / \mathrm{min} / 1.73 \mathrm{~m} 2$ $(p<0.001)$ were independently associated with starting dialysis within 30 days of $\mathrm{ASCT}^{42}$. The risk of dialysis increased exponentially with increasing the number of risk factors present. If none or 1 factor was present, risk of dialysis was $2 \%$ and $10 \%$, respectively ${ }^{42}$. Nonetheless, the presence of both, hypoalbuminemia and eGFR $<40 \mathrm{~mL} / \mathrm{min}$ resulted in a $44 \%$ risk of dialysis. As such, screening with serum albumin and eGFR may reduce the risk ${ }^{42}$. Unfortunately, these two risk factors are also risk factors for progression to ESRD as part of the natural progression of $\mathrm{AL}$ amyloidosis. Thus, the decision on whether to proceed to ASCT is a difficult one. It is important to note though, that patients are likely to experience improved renal function as the disease burden decreases post induction, then making them eligible for subsequent treatment with ASCT.
In summary, HDM-ASCT is a durable and reliable treatment option that allows for deep and sustainable responses in a minority of select, eligible patients which could be preceded by bortezomib-based induction therapy when BMPC $>10 \%$ or a foreseeable delay in ASCT is expected. Despite its consistent results, treatment with ASCT is associated with a handful of detrimental adverse events including arrhythmias, worsening HF and progression to ESRD, with the introduction of novel agents, such as bortezomib, resulting in improved results and offering an alternate treatment modality.

\section{Treatment of ASCT-ineligible patients}

The survival pattern for transplant ineligible patients has been improving over time. Trends in presentation, management and outcome among 1551 newly diagnosed AL amyloidosis patients between 2000 and 2014 were evaluated and when stratified into three eras (2000-2004, 2005-2009, and 2010-2014), survival improved significantly over time with OS rates of $25 \%, 46 \%$, and $47 \%$, respectively, $(p<0.001)^{43}$.

The main first-line options for treatment of transplant ineligible patients are (1) bortezomib-based regimens: $\mathrm{Vd}$, cyclophosphamide-bortezomib-dexamethasone (CyBorD), and bortezomib-melphalan-dexamethasone (BMDex), or (2) melphalan-dexamethasone (MDex) (Fig. 2). MDex was the first standard-intensity regimen to produce a meaningful $\mathrm{HR}$ and was the reason for the aforementioned improved survival from 2000 to 2014. The non-ASCT first-line regimen changed over time with $65 \%$ of patients in 2010-2014 receiving bortezomib-based therapy, $79 \%$ of patients in 2005-2009 receiving MDex, and 64\% of patients in 2000-2004 receiving melphalan-prednisone ${ }^{43}$. The rate of better than VGPR was higher in more recent periods (66\% vs $58 \%$ vs $51 \% ; p=0.001$ ), a change largely driven by improved VGPR rates in the non-ASCT population ${ }^{43}$. In one study by Palladini et al., $0.22 \mathrm{mg} / \mathrm{kg}$ of melphalan plus $40 \mathrm{mg} /$ day of dexamethasone were given on days $1-4$ in a 28 -day cycle ${ }^{44}$. The study proved that this combination resulted in high response rates, with a $67 \% \mathrm{HR}$ including $33 \% \mathrm{CR}$. Responses were durable, and the median time to response was 4.5 months $^{44}$. This makes MDex an effective regimen, which can be used when bortezomib is contraindicated or is unavailable. However, when dealing with elderly patients or those with pre-existing severe renal or cardiac involvement, a lower dose of dexamethasone should be considered (i.e., $20 \mathrm{mg}$ on days 1-4) due to the toxicities and increased fluid retention associated with dexamethasone.

Multiple studies have looked into the use of bortezomib-based regimens upfront ${ }^{44-46}$. Three studies, which highlight the durable HR associated with bortezomib-based regimens, are the European CyBorD collaboration study of 230 patients between 2013 and $2016^{44}$, a prospective observational study by the UK 
National Amyloidosis Center of 915 patients between 2010 and $2017^{45}$, and a phase III trial of MDex versus BMDex between 2011 and $2016^{46}$. Across these studies the CR rates on intention-to-treat (ITT) analysis ranged from 21 to $25 \%{ }^{44-46}$. Cardiac response varied from 17 to $38 \%$ and renal response, 15 to $44 \%^{44-46}$. The median OS across these studies was $>4$ years and the median follow-up period ranged from 25 to 32 months ${ }^{44-46}$. The phase III trial (NCT01277016) comparing MDex to BMDex in newly diagnosed AL patients reported very exciting data in 109 patients recruited in Europe and Australia (56 in MDex arm, 53 in BMDex arm $)^{46}$. Although there was no difference in the CR and partial response (PR) rates, or in cardiac and renal response rates, at the end of treatment after a median of five cycles, the overall HR rate was significantly better in the BMDex arm compared with the MDex arm (79\% versus $52 \%, p=0.002$ ) whereas VGPR or CR was achieved in $64 \%$ of the patients on the BMDex arm versus $39 \%$ of those on the MDex $\operatorname{arm}^{46}$. This translated into improvements with BMDex over MDex in PFS and OS with a 2-fold decrease in mortality (HR 0.5, $[0.27-0.90])^{46}$.

With respect to toxicity management, it is important to avoid bortezomib in the setting of pre-existing neuropathy or to opt for an attenuated dose regimen. Subcutaneous administration is recommended at weekly dosing; twice weekly dosing is not recommended and is likely to result in termination of therapy. Because bortezomib can be cardiotoxic to this patient population, initiating at a lower dose $\left(0.7-1.0 \mathrm{mg} / \mathrm{m}^{2}\right)$ and uptitrating as tolerable can be considered when dealing with patients with cardiac involvement. Patients should be monitored carefully for side effects and symptoms including regular monitoring for cardiac biomarkers, especially in the setting of high-risk disease. In the emergence of neuropathy or other major toxicity, the dose of bortezomib can be reduced or discontinued.

The recent ANDROMEDA phase III trial (NCT03201965) compared CyBorD to daratumumab-CyBorD in patients with newly diagnosed AL amyloidosis. Patients received weekly daratumumab in cycles 1 and 2, every 2 weeks in cycles 3-6, and every 4 weeks thereafter up to 2 years. The safety run-in of the study showed an overall HR rate of $96 \%$ without any new safety concerns beyond that already demonstrated for daratumumab in multiple myeloma (MM) and $\mathrm{CyBorD}^{47}$. The primary results of the recently completed trial indicated that the addition of daratumumab results in higher HR (92\% versus 77\%) and VGPR/CR (79\% versus $49 \%$ ) with better cardiac ( $42 \%$ versus $22 \%$ ) and renal (54\% versus $27 \%$ ) responses and prolonged PFS. Thus, the study concluded that the addition of daratumumab to CyBorD results in both, deeper and more rapid, $\mathrm{HR}^{47}$. The preliminary ANDROMEDA results are thus promising and favorably comparable to ASCT.

A phase II, open-label, dose selection study (NCT04304144) evaluated the safety and tolerability of
CAEL-101 in AL amyloidosis to provide a recommended dose of CAEL-101 to be given in combination with CyBorD for a planned randomized study in Mayo stage IIIa and IIIb patients ${ }^{48}$. CAEL-101 is an AL amyloid fibril reactive IgG1 antibody aimed at potentially clearing amyloid deposits ${ }^{48}$. The study enrolled 13 patients $(7$ heart, 3 kidney, 3 both) in a $3+3$ dose escalation safety study whereby the maximum dose tolerated was $1000 \mathrm{mg} / \mathrm{m}^{2}$ weekly for 4 weeks and then every other week. Patients were receiving CyBorD in tandem ${ }^{48}$. Of the six patients receiving the maximum dose, it was well tolerated with the exception of three significant adverse events (recurrent atrial fibrillation, Clostridium difficile infection and enlarged pleural effusion $)^{48}$. Seven patients were evaluated for organ response and 2 met criteria in the $500 \mathrm{mg} / \mathrm{m}^{2}$ cohort $^{48}$. Given the potential for early organ response, CAEL-101 could prove groundbreaking, awaiting two recently initiated phase III trials employing the maximum dose of CAEL-101 at $1000 \mathrm{mg} / \mathrm{m}^{2}$.

\section{Assessing treatment response}

Palladini et al. identified the criteria for both, hematological and cardiac responses to treatment offering surrogate end points for clinical trials ${ }^{49}$ (Fig. 3). The analysis of a multicenter cohort of 816 patients demonstrated the strong correlation between the extent of FLC reduction and survival improvement, as early as 3 months posttreatment initiation ${ }^{49}$. Four levels of response/survival were identified: $C R$ with negative serum protein electrophoresis and immunofixation (SPEP/IFE), negative urine protein electrophoresis and immunofixation (UPEP/IFE) and normal FLC ratio (FLCr); VGPR with a dFLC $<40 \mathrm{mg} / \mathrm{L}$; PR with dFLC decrease $>50 \%$; and no response (NR) with a dFLC less than that achieved in $\mathrm{PR}^{49}$. With the availability of highly effective antiplasma cell therapy, the criteria requirement of normal $\mathrm{FLCr}$ was further clarified to include abnormal FLCr inverted in favor of the nonamyloidogenic $\mathrm{FLC}^{50}$. As such, an abnormal FLCr does not preclude achieving CR when the uninvolved (uFLC) is greater than the involved FLC (iFLC) $)^{50}$.

In assessing treatment effectiveness, the target response for most patients should be at least a VGPR. In those with a low presenting dFLC of $20-50 \mathrm{mg} / \mathrm{L}$, a target response should be a dFLC $<10 \mathrm{mg} / \mathrm{L}$. If a patient has a serum M-spike (true for $\sim 50 \%$ of patients), this can help in assessing response, especially in those with a low level of circulating light chains where assessing response becomes very challenging. A retrospective analysis of 716 patients, $73 \%$ of which had a measurable M-spike before ASCT, found a measurable Mspike prior to ASCT an independent negative predictor of $\mathrm{OS}$ and $\mathrm{PFS}^{51}$. In patients with renal failure where the free light chains are cleared by the kidneys, it is sometimes hard to assess the response and a bone marrow biopsy may help to see if a deep remission has been achieved. 


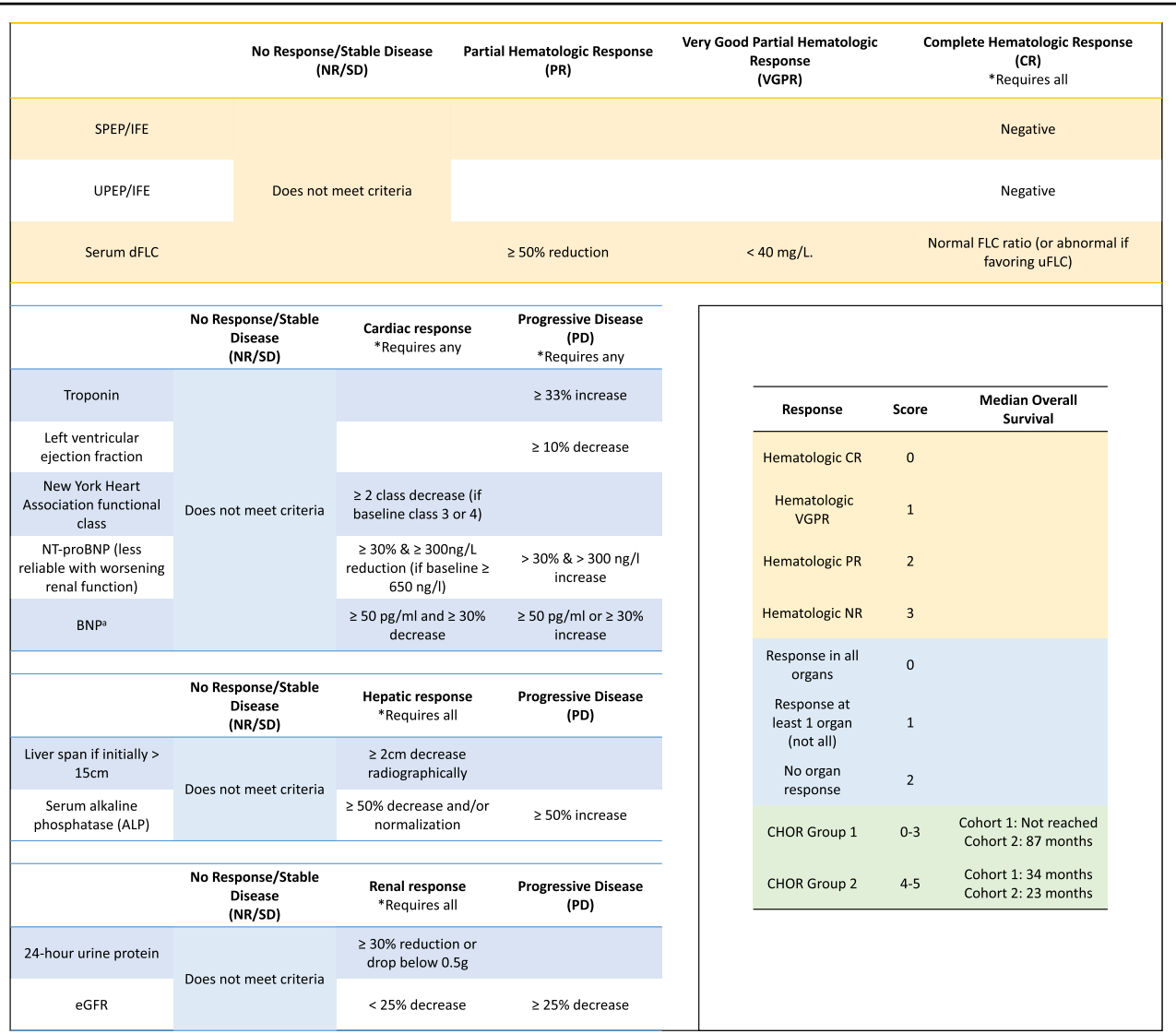

Fig. 3 Criteria for hematologic and organ response in amyloidosis. SPEP/IFE serum protein electrophoresis and immunofixation, UPEP/IFE urine protein electrophoresis and immunofixation, dFLC delta free light chain, UFLC uninvolved free light chain, eGFR estimated glomerular filtration rate, CHOR composite hematologic/organ response model. Response criteria were derived from Palladini et al. J. Clin. Oncol 2012; Comenzo et al. Leukemia 2012; and Palladini et al. Blood 2014. a: New BNP-based cardiac criteria were derived from Lilleness et al. BJH 2020. CHOR scoring model derived from Sidana et al. Blood Cancer Journal 2020.

That said, it is empirical to determine when a shift in gears and initiation of new therapy is warranted. There are three occasions when this should be considered: (1) if there is no change in serum iFLC after the first cycle, (2) if a patient does not achieve a PR after $2-3$ cycles, and (3) if treatment is not tolerated.

Increasing data are emerging about the depth of response irrespective of the HR criteria. The UK prospective study on upfront bortezomib therapy reported outcomes in the largest AL amyloidosis cohort treated with upfront bortezomib and explored the impact of posttreatment $\mathrm{dFLC}<10 \mathrm{mg} / \mathrm{L}$ ("stringent $\mathrm{dFLC}$ response") and portrayed that patients with stringent $\mathrm{dFLC}$ responses had significantly better OS and time-to-next treatment (TNT) compared to those with weaker responses ${ }^{45}$. The study looked at three levels of dFLC $(<10 \mathrm{mg} / \mathrm{L}$, $10-40 \mathrm{mg} / \mathrm{L}$, and $>40 \mathrm{mg} / \mathrm{L})^{45}$. The median OS was not reached in patients with the first two levels and was 53 months in those with dFLC of $>40 \mathrm{mg} / \mathrm{L}(\log -\operatorname{rank} p<$ $0.0001)^{45}$. The median TNT was not reached in the lowest level of dFLC, and was 38 and 13 months in the $10-40 \mathrm{mg} / \mathrm{L}$ and $>40 \mathrm{mg} / \mathrm{L}$ groups, respectively (log-rank $p<0.0001)^{45}$. Cardiac responses were better in those with stringent dFLC responses (61\%) compared with lesser responses $(45 \% ; p=0.005)^{45}$. The authors concluded that a stringent dFLC response predicted prolonged TNT and impressive organ responses ${ }^{45}$. Similarly, in another study of patients who achieved at least a VGPR, an iFLC < $20 \mathrm{mg} / \mathrm{L}$ was associated with significantly better OS and PFS $(p<0.001)^{52}$. Using a more stringent dFLC of $5 \mathrm{mg} / \mathrm{L}$, there appeared to be a further significant improvement in PFS $(p<0.001)$ but not in OS $(p=0.11)^{52}$. In summary, as well as using the HR criteria, a further understanding of the depth of response in a patient is possible if low levels of iFLC and AFLC are achieved.

It is important to emphasize that complete HR does not necessarily translate into organ response. As such, increasing attention is now being channeled to minimal residual disease (MRD). Sidana et al. evaluated MRD in 44 $\mathrm{AL}$ amyloidosis patients using MRD by next-generation 
flow-cytometry (NGF) at a minimum sensitivity of $10^{-553}$. The MRD negative rate among patients in CR was $75 \%$ relative to $50 \%$ in patients achieving VGPR. Patients with MRD-negativity were more likely to have achieve cardiac response $(67 \%$ vs $22 \%, p=0.04)$ and improved 1 -year PFS after a median follow-up of 14 months $(100 \%$ vs $64 \%, p=$ $0.006)^{53}$. Similarly, Palladini et al. evaluated MRD by NGF at a minimum sensitivity of $10^{-5}$ in $92 \mathrm{AL}$ amyloidosis patients who have achieved $\mathrm{CR}^{54}$. Fifty-four percent had detectable MRD. Undetectable MRD was associated with higher rates of renal and cardiac responses $(90 \%$ vs $62 \%$, $p=0.006$ and $95 \%$ vs $75 \%, p=0.023$, respectively) ${ }^{54}$. Even more importantly, hematological progression was more frequent in the setting of persistent MRD ( $0 \%$ vs $25 \%$ at 1 year, $p=0.001$ ), highlighting an important population who would benefit from further treatment ${ }^{54}$.

\section{Treatment of relapsed/refractory AL amyloidosis}

Upon recognizing patients who were not able to attain a satisfactory response, it becomes vital to initiate secondline therapy as soon as possible. Currently, the recommendation for second-line therapy for relapsed/refractory AL amyloidosis (RRAL) is daratumumab or daratumumab-based therapy (Fig. 2). This is due to its association with an excellent ORR ranging from 63 to $100 \%$ whereby most responses were at least VGPR with a median time to HR of 1 week and a 2-year OS rate of $74 \%^{51,55-58}$. In a recent French study including 15 patients (median age 60 years) treated with daratumumab, ORR was $86 \%$ including $43 \% \mathrm{CR}$ and $14 \% \mathrm{VGPR}^{59}$. The most common side effect was infections due to induced hypoglobulinemia $^{59}$. Thus, daratumumab has proven an excellent agent for AL amyloidosis resulting in deep remissions, high response rates and low toxicity. Nonetheless, the long-term results of daratumumab treatment and length of remission are not yet known.

Beyond daratumumab, the recommendations for thirdline treatment of RRAL depend on whether the patient had a hematological relapse $\geq 2$ years since the last therapy, in which case, the physician should consider repeating the original therapy. If the patient is not bortezomib refractory, then a bortezomib-based regimen is the preferred choice (CyBorD, BMDex, or Vd). On the other hand, if the patient is bortezomib refractory, then pomalidomide-dexamethasone (pom-dex) or lenalidomide-dexamethasone (len-dex) can be used. Importantly, ASCT can be considered in the relapsed setting, either as first ASCT or as a second transplant.

IMiDs are generally not well tolerated by $\mathrm{AL}$ amyloidosis patients. Whereby thalidomide is known for its high toxicity, lenalidomide comes with concerns too. The two main toxicity concerns with lenalidomide are (1) lenalidomide-related renal deterioration which, in a study of 41 patients, occurred in $66 \%$ and was only reversible in about half of them ${ }^{60}$, and (2) a rise in NT-proBNP/BNP. To avoid or manage this type of toxicity, we recommend a low starting dose of lenalidomide of $5-15 \mathrm{mg}$ /day with the lower dose reserved for elderly patients or patients with baseline cardiac involvement or elevated creatinine. The dose can then be adjusted according to tolerability. One should observe carefully for fluid retention by weighing patients daily and measuring cardiac biomarkers as well as monitoring kidney function.

Pomalidomide is slightly better tolerated than lenalidomide and several studies have demonstrated rapid responses (median 1-3 months) and improved survival with pom-dex. A study of 33 patients with a median follow-up of 28 months highlighted the activity of the pom-dex combination even in the setting of having failed lenalidomide and bortezomib whereby the confirmed overall HR rate and PFS were $48 \%$ and $14 \%$, respectively ${ }^{61}$. Median time to response was 1.9 months and the median duration of response was 19 months ( $95 \% \mathrm{CI}, 8.3$ to not attained $[\mathrm{NA}])^{61}$. Similarly, a phase $1 / 2$ trial of pom-dex reported an ORR of $50 \%$ in 24 evaluable patients ${ }^{62}$. The median time to best ORR was three cycles, and median duration of ORR was 15 months ${ }^{62}$. With a median followup of 17.1 months, median OS had not yet been reached, and median event-free survival (EFS) was 17.8 months ${ }^{62}$. A phase 2 trial of pom-dex rescue treatment in 28 patients previously exposed to alkylators, PIs, and lenalidomide demonstrated a hematological ORR in $68 \%$ and a VGPR/ CR in 29\%, as well as improved survival with median time to response of 1 month $^{63}$. Hematologic ORRs were also rapid, with half of responding patients doing so within 2 months ${ }^{63}$. Pomalidomide dose reduction was required in two out of three of those studies due to toxicity whereby patients experienced grade 3-4 myelosuppression $(26-45 \%)$, fatigue (18\%), pneumonia $(11-21 \%)$, renal failure $(3-7.5 \%)$, and arrhythmias $(0-21 \%)^{61,63}$. It is important to note that $\mathrm{AL}$ amyloidosis patients treated with pomalidomide frequently experience increases in proBNP/BNP without clinical congestive heart failure.

PIs other than bortezomib also play a role. A few studies have looked at treatment of AL amyloidosis with the second-generation PIs, ixazomib and carfilzomib. The phase III randomized controlled trial TOURMALINEAL1 (NCT01659658) has recently published its results. It compared the combination of ixazomib and dexamethasone (ixa-dex, $n=85)$ to physician choice $(n=83$ : len-dex, $n=47$; melphalan-dex, $n=24 ; \quad$ cyclophosphamide-dex, $n=10 ; \quad$ thalidomide-dex, $n=2)^{64}$. TOURMALINE-AL1 is the first phase III trial in patients with RRAL to show a significant clinical outcome improvement. Despite the trial not meeting the first primary endpoint of hematologic ORR, treatment with ixadex resulted in an improved CR rate (26\% versus $19 \%)$ and duration of overall HR (46.5 months versus 20.2 months) 
when compared to physician's choice of treatment ${ }^{64}$. Although there was no difference between OS and overall PFS, treatment with ixa-dex increased hematologic/vital organ PFS and decreased time to vital organ deterioration/death, time to treatment failure, and time to subsequent therapy ${ }^{64}$. Considering that the duration of treatment in the ixa-dex arm was twice that of the physician's choice arm (median 11.7 months versus 4.9 months), continuous ixa-dex was generally well tolerated $^{64}$. When stratified by prior PI exposure, hematologic ORR was $63 \%$ versus $50 \%$ for Ixa-dex versus physician choice in PI-naïve patients and $41 \%$ versus $51 \%$ in PI-exposed ${ }^{65}$. Thus, ORR was higher for Ixa-dex versus physician choice in PI-naive patients and lower in PIexposed (though not statistically significant) ${ }^{65}$. Based on these results, ixa-dex may be considered a new option for patients with RRAL, given sensitivity to bortezomib. Ixazomib was also studied in the all-oral combination, ixazomib, cyclophosphamide, and dexamethasone in a phase II trial ${ }^{66}$. The study included 35 patients with newly diagnosed, biopsy-proven AL amyloidosis but excluded patients with severe organ involvement (alkaline phosphatase $>750 \mathrm{U} / \mathrm{L}, \mathrm{CrCl}<30 \mathrm{~mL} / \mathrm{min}$, or NT-proBNP $\geq$ $7500 \mathrm{ng} / \mathrm{dL})^{66}$. The overall HR was $57 \%$ including $\mathrm{CR}$ in $4 \%$, VGPR in $26 \%$, and PR in $17 \%{ }^{66}$. Median PFS and OS have not been reached, four patients had disease progression and six have $\operatorname{died}^{66}$. Thus far, this points to modest activity in the all-oral regimen awaiting further results.

On the other hand, carfilzomib is a challenging agent due to the high incidence of cardiac involvement with $\mathrm{AL}$ amyloidosis. It is known that up to $10 \%$ of MM patients treated with carfilzomib experience cardiac toxicity. It is also an intravenous infusion and patients require hydration, which can aggravate patients predisposed to congestive heart failure. Results of an investigator-initiated, multicenter, phase I/II study of carfilzomib in AL amyloidosis (NCT01789242) showed that carfilzomib monotherapy was feasible and effective in 28 RRAL patients ${ }^{67}$. The dose escalating phase identified a maximum tolerated dose of 20/ $36 \mathrm{mg} / \mathrm{m}^{2}$ (which is lower than that for MM) ${ }^{67}$. HR rates were promising in this bortezomib-exposed population, including PI-refractory patients. The ORR and $\geq$ VGPR were $54 \%$ and 39\%, respectively, and although these are encouraging results, cardiac toxicity was experienced by $36 \%$ (including major toxicities such as ventricular tachyarrhythmia, decrease in EF, and hypoxemia) ${ }^{67}$.

Venetoclax is an oral, small-molecule B-cell lymphoma 2 (BCL-2) inhibitor that induces cellular apoptosis, with encouraging activity in chronic lymphoid leukemia (CLL), non-Hodgkin lymphoma (NHL), acute myeloid leukemia (AML), and a subset of MM patients whose clonal plasma cells harbor $\mathrm{t}(11 ; 14)$ and/or overexpress BCL-2. Venetoclax may be useful in the management of $\mathrm{AL}$ amyloidosis for two reasons: $\mathrm{t}(11 ; 14)$ is present in $\sim 50 \%$ of $\mathrm{AL}$ amyloidosis patients ${ }^{17}$, and patients with this translocation are less likely to respond to a bortezomib-based regimen ${ }^{17,18}$. There is limited experience with venetoclax in AL amyloidosis but in 2018, Leung et al. reported on perhaps the first patient with AL amyloidosis to be successfully treated with venetoclax after his disease markers plateaued on $\mathrm{CyBorD}^{18}$. Sidiqi et al. have recently demonstrated efficacy and safety of venetoclax in 12 RRAL patients treated with venetoclax between January 2017 and May 2019 ${ }^{68}$. Patients had a median of two prior lines and all but 1 had $t(11 ; 14)^{68}$. The dose used was $400-800 \mathrm{mg} / \mathrm{d}$; 7 in combination with dexamethasone ${ }^{68}$. Eight patients were evaluable for response and the response rate was $87 \%$ comprising 3 with CR and 4 with $\mathrm{VGPR}^{68}$. The median time to best response was 3.4 months and median follow-up was 11.5 months, but two patients progressed at 4 and 5 months from initiation of therapy $^{68}$. Toxicity was minimal and there were no reports of tumor lysis syndrome. Notably, in this cohort no deaths have been observed so far. Albeit a retrospective case series with a small sample size, this study suggests high efficacy and good tolerability of venetoclax monotherapy and combination therapy in patients harboring $t(11 ; 14)$.

Bendamustine is another agent to consider although the response rates are not very high in the $\mathrm{AL}$ amyloidosis population. However, for those with an IgM amyloidosis or a lymphoplasmacytic histology, in combination with rituximab, response rates are much better, and survival is improved even in heavily pretreated patients. This is highlighted by three recently published studies ${ }^{69-71}$. Milani et al. reported on 122 patients, 36 of whom had IgM amyloidosis ${ }^{69}$. With a median time to response of 3 months, the ORR to bendamustine in the whole cohort was $35 \%{ }^{69}$. In the 12 patients with IgM-AL amyloidosis, this was achieved in $58 \%$ of subjects $(21 \% \text { with } \geq \text { VGPR })^{69}$. The median follow-up of living patients was 31 months (IQR, 17-46) and severe adverse effects were observed in $26 \%{ }^{69}$. Lentzsch et al. conducted a phase II, multicenter trial (NCT01222260) to assess the efficacy and safety of bendamustine with dexamethasone in 31 patients with persistent or progressive AL amyloidosis after $\geq 1$ prior therapy ${ }^{70}$. Dose reduction occurred in $31 \%$ of patients and $57 \%$ of patients achieved a PR or better $(11 \%$ CR, $18 \%$ VGPR $)^{70}$. The median OS was 18.2 months and the median PFS was 11.3 months $^{70}$. Side effects were commonly myelosuppression, fatigue, and nausea $/$ vomiting $^{70}$. Manwani et al. reported outcomes in 27 patients identified from the UK National Amyloidosis Centre database (5 of which were RRAL) treated with bendamustine and rituximab showing an ORR of 59\% (11\% CR, 37\% VGPR, and $11 \% \mathrm{PR})^{71}$. The median follow-up was 18 months and the median PFS was 34 months. Side effects comprised: GI symptoms, fatigue, cytopenias, and neutropenic fever $^{71}$. 
Finally, another anti-CD38 monoclonal antibody, isatuximab is an IgG1k monoclonal antibody with highaffinity binding to CD38 expressed on plasma cells, which has proven efficacious in $M M$ as a single agent or in combination. The phase II trial SWOG S1702 (NCT03499808) investigates the role of isatuximab. It included 36 patients with RRAL who have received at least one previous line of therapy ${ }^{72}$. The median age was 70 and prior therapies included PI (89\%), high-dose therapy followed by ASCT (47\%), IMiDs (28\%) and an anti-CD38 monoclonal antibody $(6 \%)^{72}$. Nineteen patients discontinued treatment whereby the most common reasons were adverse events, disease progression, suboptimal response and COVID-19 concerns ${ }^{72}$. The median duration of therapy for the 17 patients currently on treatment is 11.8 months $^{72}$. The overall HR was $77 \%$ with $3 \%$ CR, $54 \%$ VGPR, and $20 \% \mathrm{PR}^{72}$. The majority of drug-related side effects were grade I/II infusion-related (50\%), grade I anemia $\left(25 \%\right.$ and lymphopenia $(22 \%)^{72}$. Isatuximab thus has promising efficacy with a side effect profile similar to other anti-CD38 monoclonal antibodies ${ }^{72}$.

\section{Conclusion}

The presentation of AL amyloidosis could be rather deceitful due to its mimicry of various conditions. The hallmark of disease management is early diagnosis, ideally during the routine monitoring phase of high-risk patients before the onset of symptoms and irreversible organ damage. The goal of therapy in this challenging disease is at least a hematological VGPR $(\mathrm{dFLC}>50 \mathrm{mg} / \mathrm{L})$. Treatment should be guided by ASCT-eligibility given the robust and durable response attainable with transplant. This could be preceded by induction chemotherapy with bortezomib-based regimens especially in the setting of high disease burden or foreseeable delay in ASCT. In the setting of ASCT-ineligibility, upfront treatment with bortezomib-based regimens, now with the incorporation of daratumumab is standard. In the setting of relapse, the best response appears to be with the use of daratumumab though multiple emerging data on other novel agents including ixazomib, isatuximab, and pomalidomide are quite promising and remarkable. As we await the results of the multiple ongoing trials including the recently initiated trials involving the anti-fibril, CAEL-101, the field is still in dire need of research involving higher risk patients.

\footnotetext{
Author details

'Department of Internal Medicine, Jacobi Medical Center, Albert Einstein College of Medicine, New York, NY, USA. 'Department of Internal Medicine, American University of Beirut, Beirut, Lebanon. ${ }^{3}$ Department of Clinical Hematology and Cellular Therapy, Saint-Antoine Hospital, AP-HP, Sorbonne University, and INSERM, Saint-Antoine Research Centre, Paris, France. ${ }^{4}$ Institut de Cancerologie de l'Ouest, Centre René Gauducheau, Nantes-St Herblain, France
}

Conflict of interest

The authors declare no competing interests.

\section{Publisher's note}

Springer Nature remains neutral with regard to jurisdictional claims in published maps and institutional affiliations.

Received: 23 February 2021 Revised: 11 April 2021 Accepted: 27 April 2021 Published online: 18 May 2021

\section{References}

1. Kyle, R. A. et al. Incidence and natural history of primary systemic amyloidosis in Olmsted County, Minnesota, 1950 through 1989. Blood 79, 1817-1822 (1992).

2. Palladini, G., Milani, P. \& Merlini, G. Novel strategies for the diagnosis and treatment of cardiac amyloidosis. Expert. Rev. Cardiovasc. Ther. 13, 1195-1211 (2015).

3. Obici, L., Perfetti, V., Palladini, G., Moratti, R. \& Merlini, G. Clinical aspects of systemic amyloid diseases. Biochim. Biophys. Acta 1753, 11-22 (2005).

4. Blake, C. C., Geisow, M. J., Oatley, S. J., Rerat, B. \& Rerat, C. Structure of prealbumin: secondary, tertiary and quaternary interactions determined by Fourier refinement at 1.8 A. J. Mol. Biol. 121, 339-356 (1978).

5. Monaco, H. L., Rizzi, M. \& Coda, A. Structure of a complex of two plasma proteins: transthyretin and retinol-binding protein. Science 268, 1039-1041 (1995).

6. Colon, W. \& Kelly, J. W. Partial denaturation of transthyretin is sufficient for amyloid fibril formation in vitro. Biochemistry 31, 8654-8660 (1992).

7. Maurer, M. S. et al. Tafamidis treatment for patients with transthyretin amyloid cardiomyopathy. N. Engl. J. Med. 379, 1007-1016 (2018).

8. Merlini, G. \& Palladini, G. Differential diagnosis of monoclonal gammopathy of undetermined significance. Hematol. Am. Soc. Hematol. Educ. Program. 2012, 595-603 (2012).

9. Palladini, G., Milani, P. \& Merlini, G. Management of AL amyloidosis in 2020. Hematol. Am. Soc. Hematol. Educ. Program. 2020, 363-371 (2020).

10. Muchtar, E. et al. Staging systems use for risk stratification of systemic amyloidosis in the era of high-sensitivity troponin T assay. Blood 133, 763-766 (2019).

11. Dispenzieri, A. et al. Serum cardiac troponins and N-terminal pro-brain natriuretic peptide: a staging system for primary systemic amyloidosis. J. Clin. Oncol. 22, 3751-3757 (2004).

12. Wechalekar, A. D. et al. A European collaborative study of treatment outcomes in 346 patients with cardiac stage III AL amyloidosis. Blood 121, 3420-3427 (2013).

13. Kumar, S. et al. Revised prognostic staging system for light chain amyloidosis incorporating cardiac biomarkers and serum free light chain measurements. J. Clin. Oncol. 30, 989-995 (2012).

14. Lilleness, B., Ruberg, F. L., Mussinelli, R., Doros, G. \& Sanchorawala, V. Development and validation of a survival staging system incorporating BNP in patients with light chain amyloidosis. Blood 133, 215-223 (2019).

15. Muchtar, E. et al. A modern primer on light chain amyloidosis in 592 patients with mass spectrometry-verified typing. Mayo Clin. Proc. 94, 472-483 (2019).

16. Ozga, M. et al. The effect of cytogenetic abnormalities on organ involvement and survival in patients with AL amyloidosis. Blood 134, 1833-1833 (2019).

17. Bochtler, T. et al. Translocation $t(11 ; 14)$ is associated with adverse outcome in patients with newly diagnosed $\mathrm{AL}$ amyloidosis when treated with bortezomib-based regimens. J. Clin. Oncol. 33, 1371-1378 (2015).

18. Leung, N., Thome, S. D. \& Dispenzieri, A. Venetoclax induced a complete response in a patient with immunoglobulin light chain amyloidosis plateaued on cyclophosphamide, bortezomib and dexamethasone. Haematologica 103, e135-e137 (2018).

19. Gatt, M. E. \& Palladini, G. Light chain amyloidosis 2012: a new era. Br. J. Haematol. 160, 582-598 (2013)

20. Gertz, M. et al. Troponin T level as an exclusion criterion for stem cell transplantation in light-chain amyloidosis. Leuk. Lymphoma 49, 36-41 (2008).

21. Dingli, D. et al. Stem cell transplantation in patients with autonomic neuropathy due to primary (AL) amyloidosis. Neurology 74, 913-918 (2010).

22. Gertz, M. A. et al. Trends in day 100 and 2-year survival after auto-SCT for AL amyloidosis: outcomes before and after 2006. Bone Marrow Transplant. 46 970-975 (2011). 
23. Tandon, N. et al. Revisiting conditioning dose in newly diagnosed light chain amyloidosis undergoing frontline autologous stem cell transplant: impact on response and survival. Bone Marrow Transplant. 52, 1126-1132 (2017).

24. Cibeira, M. T. et al. Outcome of AL amyloidosis after high-dose melphalan and autologous stem cell transplantation: long-term results in a series of 421 patients. Blood 118, 4346-4352 (2011).

25. Muchtar, E. et al. Ten-year survivors in AL amyloidosis: characteristics and treatment pattern. Br. J. Haematol. 187, 588-594 (2019).

26. Jaccard, A. et al. High-dose melphalan versus melphalan plus dexamethasone for AL amyloidosis. N. Engl. J. Med. 357, 1083-1093 (2007).

27. Mhaskar, R., Kumar, A., Behera, M., Kharfan-Dabaja, M. A. \& Djulbegovic, B. Role of high-dose chemotherapy and autologous hematopoietic cell transplantation in primary systemic amyloidosis: a systematic review. Biol. Blood Marrow Transplant. 15, 893-902 (2009).

28. D'Souza, A. et al. Improved outcomes after autologous hematopoietic cell transplantation for light chain amyloidosis: a Center for International Blood and Marrow Transplant Research Study. J. Clin. Oncol. 33, 3741-3749 (2015).

29. Hwa, Y. L. et al. Induction therapy pre-autologous stem cell transplantation in immunoglobulin light chain amyloidosis: a retrospective evaluation. Am. J. Hematol. 91, 984-988 (2016)

30. Afrough, A. et al. Impact of induction therapy on the outcome of immunoglobulin light chain amyloidosis after autologous hematopoietic stem cell transplantation. Biol. Blood Marrow Transplant. 24, 2197-2203 (2018).

31. Sanchorawala, V. et al. Induction therapy with bortezomib followed by bortezomib-high dose melphalan and stem cell transplantation for light chain amyloidosis: results of a prospective clinical trial. Biol. Blood Marrow Transplant. 21, 1445-1451 (2015).

32. Gupta, V. K. et al. Induction therapy with bortezomib and dexamethasone and conditioning with high-dose melphalan and bortezomib followed by autologous stem cell transplantation for immunoglobulin light chain amyloidosis: long-term follow-up analysis. Biol. Blood Marrow Transplant. 25, e169-e173 (2019).

33. Minnema, M. C. et al. Bortezomib-based induction followed by stem cell transplantation in light chain amyloidosis: results of the multicenter HOVON 104 trial. Haematologica 104, 2274-2282 (2019).

34. Bashir, Q., Langford, L. A., Parmar, S., Champlin, R. E. \& Qazilbash, M. H. Primary systemic amyloid light chain amyloidosis decompensating after filgrastiminduced mobilization and stem-cell collection. J. Clin. Oncol. 29, e79-e80 (2011).

35. Yeh, J. C., Shank, B. R., Milton, D. R. \& Qazilbash, M. H. Adverse prognostic factors for morbidity and mortality during peripheral blood stem cell mobilization in patients with light chain amyloidosis. Biol. Blood Marrow Transplant. 24, 815-819 (2018).

36. Sanchorawala, V. High-dose melphalan and autologous peripheral blood stem cell transplantation in AL amyloidosis. Acta Haematol. 143, 381-387 (2020).

37. Oran, B. et al. Predictive factors for hematopoietic engraftment after autologous peripheral blood stem cell transplantation for AL amyloidosis. Bone Marrow Transplant. 35, 567-575 (2005).

38. Sidana, S. et al. Prognostic significance of holter monitor findings in patients with light chain amyloidosis. Mayo Clin. Proc. 94, 455-464 (2019).

39. Girnius, S. et al. Safety and efficacy of high-dose melphalan and auto-SCT in patients with $\mathrm{AL}$ amyloidosis and cardiac involvement. Bone Marrow Transplant. 49, 434-439 (2014).

40. Bleeker, J. S. et al. Evaluation of pretransplant factors predicting cardiac dysfunction following high-dose melphalan conditioning and autologous peripheral blood stem cell transplantation. Eur. J. Haematol. 89, 228-235 (2012).

41. Siddiqi, O. K. \& Ruberg, F. L. Cardiac amyloidosis: an update on pathophysiology, diagnosis, and treatment. Trends Cardiovasc. Med. 28, 10-21 (2018).

42. Leung, $\mathrm{N}$. et al. The impact of dialysis on the survival of patients with immunoglobulin light chain (AL) amyloidosis undergoing autologous stem cell transplantation. Nephrol. Dial. Transplant. 31, 1284-1289 (2016).

43. Muchtar, E. et al. Improved outcomes for newly diagnosed AL amyloidosis between 2000 and 2014: cracking the glass ceiling of early death. Blood 129, 2111-2119 (2017)

44. Palladini, G. et al. Association of melphalan and high-dose dexamethasone is effective and well tolerated in patients with $\mathrm{AL}$ (primary) amyloidosis who are ineligible for stem cell transplantation. Blood 103, 2936-2938 (2004).

45. Manwani, R. et al. A prospective observational study of 915 patients with systemic AL amyloidosis treated with upfront bortezomib. Blood 134, 2271-2280 (2019).

46. Kastritis, E. et al. Bortezomib, melphalan, and dexamethasone for light-chain amyloidosis. J. Clin. Oncol. 38, 3252-3260 (2020).
47. Kastritis, E. et al. Subcutaneous daratumumab + cyclophosphamide, bortezomib, and dexamethasone (CyBorD) in patients with newly diagnosed light chain $(\mathrm{AL})$ amyloidosis: primary results from the phase 3 ANDROMEDA study. EHA25 Virtual Congress. Abstract LB2604 (2020).

48. Khouri, J. et al. Safety, tolerability and efficacy of Cael-101 in AL amyloidosis patients treated on a phase 2, open-label, dose selection study to evaluate the safety and tolerability of Cael-101 in patients with AL amyloidosis. Blood 136, 21-21 (2020).

49. Palladini, G. et al. New criteria for response to treatment in immunoglobulin light chain amyloidosis based on free light chain measurement and cardiac biomarkers: impact on survival outcomes. J. Clin. Oncol. 30, 4541-4549 (2012).

50. Palladini, G. et al. Clarification on the definition of complete haematologic response in light-chain (AL) amyloidosis. Amyloid 28, 1-2 (2021).

51. Al Saleh, A. S. et al. Presence of a measurable M-spike before autologous stem cell transplantation is associated with shorter survival in patients with light chain amyloidosis. Blood 136, 22-23 (2020).

52. Muchtar, E. et al. Optimizing deep response assessment for $\mathrm{AL}$ amyloidosis using involved free light chain level at end of therapy: failure of the serum free light chain ratio. Leukemia 33, 527-531 (2019).

53. Sidana, S. et al. Impact of minimal residual negativity using next generation flow cytometry on outcomes in light chain amyloidosis. Am. J. Hematol. 95, 497-502 (2020).

54. Palladini, G. et al. Minimal residual disease negativity by next-generation flow cytometry is associated with improved organ response in $\mathrm{AL}$ amyloidosis. Blood Cancer J. 11, 34 (2021).

55. Kaufman, G. P. et al. Daratumumab yields rapid and deep hematologic responses in patients with heavily pretreated AL amyloidosis. Blood 130, 900-902 (2017).

56. Abeykoon, J. P. et al. Daratumumab-based therapy in patients with heavilypretreated AL amyloidosis. Leukemia 33, 531-536 (2019).

57. Khouri, J. et al. Daratumumab proves safe and highly effective in AL amyloidosis. Br. J. Haematol. 185, 342-344 (2019).

58. Sanchorawala, V. et al. Safety, tolerability, and response rates of daratumumab in relapsed AL amyloidosis: results of a phase 2 study. Blood 135, 1541-1547 (2020).

59. Van de Wyngaert, Z. et al. Daratumumab is effective in the relapsed or refractory systemic light-chain amyloidosis but associated with high infection burden in a frail real-life population. Br. J. Haematol. 188, e24-e27 (2020).

60. Specter, R. et al. Kidney dysfunction during lenalidomide treatment for AL amyloidosis. Nephrol. Dial. Transplant. 26, 881-886 (2011).

61. Dispenzieri, $\mathrm{A}$. et al. Activity of pomalidomide in patients with immunoglobulin light-chain amyloidosis. Blood 119, 5397-5404 (2012).

62. Sanchorawala, $\mathrm{V}$. et al. Pomalidomide and dexamethasone in the treatment of AL amyloidosis: results of a phase 1 and 2 trial. Blood 128, 1059-1062 (2016).

63. Palladini, G. et al. A phase 2 trial of pomalidomide and dexamethasone rescue treatment in patients with AL amyloidosis. Blood 129, 2120-2123 (2017).

64. Dispenzieri, A. et al. Primary results from the phase 3 Tourmaline-AL1 trial of ixazomib-dexamethasone versus physician's choice of therapy in patients (Pts) with relapsed/refractory primary systemic AL amyloidosis (RRAL). Blood 134, 139-139 (2019).

65. Du Bois, A. et al. Randomized phase III study to evaluate the impact of secondary cytoreductive surgery in recurrent ovarian cancer: final analysis of AGO DESKTOP III/ENGOT-ov20. J. Clin. Oncol. 38, 6000-6000 (2020).

66. Muchtar, E. et al. Phase 2 trial of ixazomib, cyclophosphamide and dexamethasone for treatment of previously untreated light chain amyloidosis. Blood 136, 52-53 (2020).

67. Manwani, R. et al. Carfilzomib is an effective upfront treatment in AL amyloidosis patients with peripheral and autonomic neuropathy. Br. J. Haematol. 187, 638-641 (2019).

68. Sidiqi, M. H. et al. Venetoclax for the treatment of translocation $(11 ; 14)$ AL amyloidosis. Blood Cancer J. 10, 55 (2020).

69. Milani, P. et al. Treatment of AL amyloidosis with bendamustine: a study of 122 patients. Blood 132, 1988-1991 (2018).

70. Lentzsch, S. et al. Bendamustine with dexamethasone in relapsed/refractory systemic light-chain amyloidosis: results of a phase II study. J. Clin. Oncol. 38, 1455-1462 (2020).

71. Manwani, R. et al. Treatment of IgM-associated immunoglobulin light-chain amyloidosis with rituximab-bendamustine. Blood 132, 761-764 (2018).

72. Parker, T. L. et al. A phase II study of isatuximab (SAR650984) (NSC-795145) for patients with previously treated AL amyloidosis (SWOG S1702; NCT\#03499808). Blood 136, 20-21 (2020). 